\title{
Timing of Surgery to Treat Ulcerative Colitis: An Investigation Focused on Japanese Adults
}

\author{
Saki Yamada Hitoshi Kameyama Kaoru Abe Kana Tanaka Hidehito Oyanagi \\ Yosuke Tajima Masato Nakano Yoshifumi Shimada Jun Sakata \\ Toshifumi Wakai
}

Division of Digestive and General Surgery, Niigata University Graduate School of Medical and Dental Sciences, Niigata, Japan

\section{Keywords}

Ulcerative colitis · Questionnaire · Quality of life · Timing of surgery

\begin{abstract}
Introduction: There are no specific standards for the timing of surgery to treat ulcerative colitis, so reaching a decision on timing is often difficult. Objective: The aim of this study was to evaluate the timing of surgery from the patients' perspective. Methods: From among 203 patients who underwent restorative proctocolectomy with ileal pouch anal anastomosis to treat ulcerative colitis at our hospital from 1985 to 2013, we mailed a questionnaire to 101 of those who are currently attending periodic follow-up at our hospital. Results: We analyzed 72 valid responses (71.2\%). Overall, 65 patients $(90.3 \%)$ responded that they were happy that they underwent surgery, 38 patients $(52.8 \%)$ responded that the timing of surgery was appropriate, and 32 patients (44.4\%) responded that they would have preferred to have had surgery earlier. The group of patients who would have preferred to have had surgery earlier included 23 patients (71.9\%) who had undergone emergency surgery; the incidence of emergency surgery was significantly higher than in the group of 13 patients (34.2\%) who had responded that
\end{abstract}

the timing of surgery was appropriate $(p=0.002)$. Scores on the Medical Outcomes Study 36-Item Short-Form Health Survey (SF36), which reflect postoperative quality of life, were maintained at the same level as the Japanese standard values in our Japanese patients. Conclusion: The degree of satisfaction of patients who underwent surgery for ulcerative colitis was favorable, although it is important to consider surgery at an earlier stage in patients who may need emergency surgery.

(c) 2020 The Author(s)

Published by S. Karger AG, Basel

\section{Introduction}

Tremendous advances have been made in the treatment of ulcerative colitis due to the development of thiopurine formulations, calcineurin inhibitors, and biologic formulations. However, reports indicate that $25-30 \%$ of patients will ultimately require surgical treatment [1-3]. In Japan, surgical indications for ulcerative colitis are divided into absolute and relative indications [4]. Absolute indications include colonic perforation, high-volume hemorrhage, and toxic megacolon, following a severe or fulminant clinical course and in which potent drug treatment is ineffective, and colon cancer or high-grade dys- 
plasia. Relative indications include intractability and difficulty continuing drug treatment due to adverse events or complications outside the gastrointestinal tract, as well as gastrointestinal complications such as stenosis or fistula. In the United States, surgical indications are almost the same: acute severe condition, intractability, and colon cancer and dysplasia [5]. The standard surgical technique in both countries is restorative proctocolectomy with ileal pouch anal anastomosis (RP-IPAA), and depending on the general condition and age of the patient, surgery may be performed at a number of stages or the patient may be fitted with a permanent stoma [4-7].

There are no concrete recommendations for the timing of surgery in either Japan or the United States, so reaching a decision on timing is often difficult. From the perspective of the healthcare professionals involved, there should be close cooperation between the internal medicine specialist and surgeon, and they should decide on the timing of surgery together $[1,4]$. In this study, we investigated the issue of timing of surgery from the patients' perspective by conducting a questionnaire survey with patients who had undergone surgery before the development of biologic drugs and so were not taking them before or after the survey.

\section{Materials and Methods}

From among 203 patients who underwent RP-IPAA to treat ulcerative colitis at our hospital from 1985 to 2013, we mailed a questionnaire to 101 of the patients who were currently attending periodic follow-up at our hospital. Among the 78 individuals who responded to the survey (response rate $77.2 \%$ ), we excluded 5 responses that did not include the identity of the responder and 1 response from a patient taking biologic drugs, leaving a total of 72 subjects (Fig. 1).

The questionnaire was created with reference to a report by Neumann et al. [1] (Fig. 2). In addition, we evaluated postoperative quality of life (QOL) using the Medical Outcomes Study 36-Item Short-Form Health Survey (SF36). The SF36 score was shown in relation to the Japanese standard value of 50 points, in accordance with the scoring manual. Statistical analysis was performed using the Mann-Whitney $U$ test for continuous variables and the $\chi^{2}$ test for categorical variables; a $p$ value of $<0.05$ was considered significant. This study was approved by the institutional review board of Niigata University (approval number 2019-0099).

\section{Results}

Table 1 shows the patient characteristics. As a rule, we performed surgery in 3 stages and created a $\mathrm{W}$-shaped ileal pouch. A total of 65 patients $(90.3 \%)$ responded that

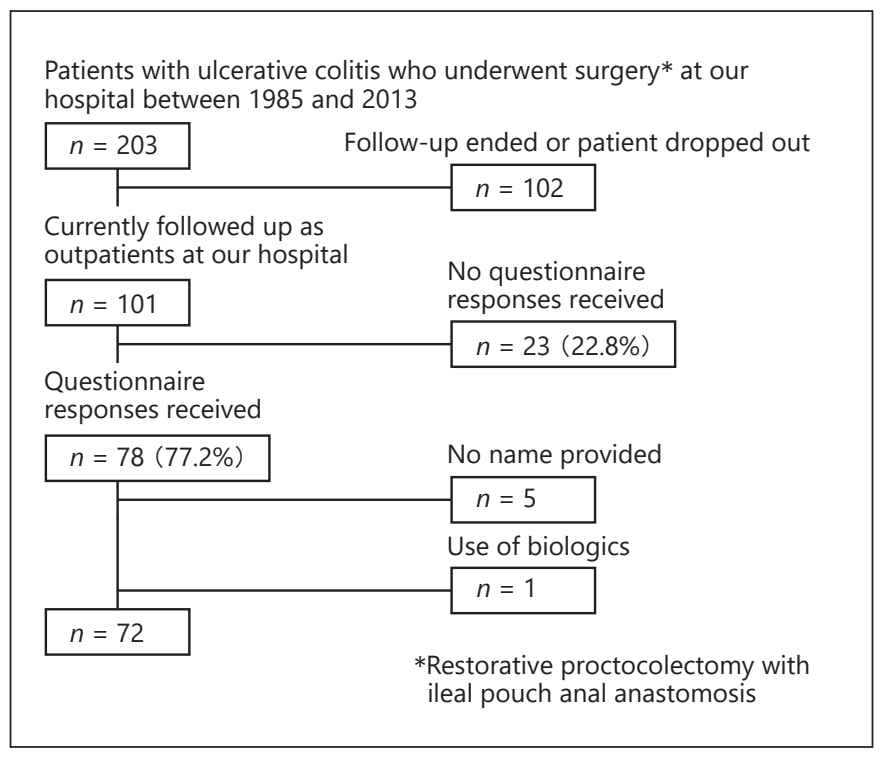

Fig. 1. Subjects in this study.

Table 1. Patient characteristics
Men/women, $n$

Age at time of survey, years

J-shaped/W-shaped ileal pouch, $n$

II/III-stage surgery, $n$

Elective surgery/(semi-)emergency surgery, $n$

Surgical indication, $n$

Relative indication/absolute indication

(including cases of cancer)

Postoperative follow-up observation, months

Period from onset until IPAA, months

Total steroid dose, $\mathrm{mg}$

Preoperative steroid dose/day, mg
$35 / 37$

$50(18-75)$

$10 / 62$

$19 / 53$

$32 / 40$

$35 / 37(10)$

$96(14-348)$

$53(1-300)$

$10,000(0-90,000)$

$25(0-120)$
Values are medians (ranges) unless otherwise indicated. IPAA, ileal pouch anal anastomosis.

they were happy that they had surgery, and none of the patients responded that they wished that they had not had surgery (Fig. 3a). Figure 3b shows patient opinions about the timing of surgery. Overall, 38 patients $(52.8 \%)$ responded that the timing of surgery was just right (appropriate group), 32 patients (44.4\%) responded that they would have preferred to have had surgery earlier (earlier group), and 2 patients (2.8\%) responded that they would have preferred to have had surgery later (later group). The earlier group stated they would have preferred to have had surgery a median period of 6 months earlier; their reasons are shown in Figure 4. The most common reason was "I 
Q1. What did you think when you actually had the operation?

I was glad that I had the operation.

I didn't think anything about it.

I wish that I hadn't had an operation.

Q2. What did you think about the timing of your operation?

I would have preferred an earlier operation. (By how many months? _months)

The timing was just right.

I would have preferred a later operation. (By how many months? _months)

(1) Please answer the following if you answered that you would have preferred an earlier operation. Please mark the applicable reasons with a check mark. Multiple responses are allowed.

- I was better able to control defecation.

$\square$ I had less blood in my stool (hematochezia).

- My pain improved.

I I had fewer problems socially (going outside, travel, school, eating out with friends, etc.).

$\square$ Working was easier.

I was concerned about what would happen after surgery, but it was alright.

- My general condition gradually worsened, so I wanted to have the operation before things became really bad.

$\square$ I suffered for a long time because of intensive drug treatment.

(2) Please answer the following question if you answered above that you would have preferred to have had your operation later. Which of the following reasons apply?

$\square$ I have problems that I didn't have before my operation.

$\checkmark$ I believe there is a better form of treatment that was not available when I had my operation.

Fig. 2. Questionnaire items asked in this study, which were designed based on Neumann et al. [1].

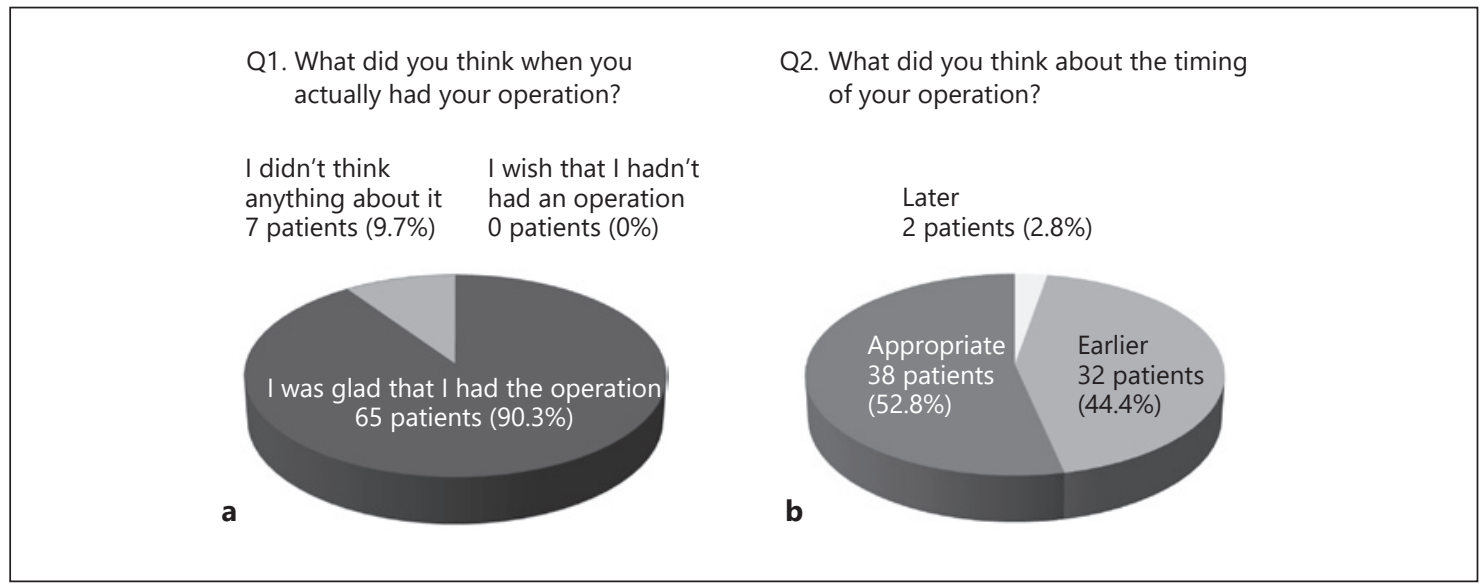

Fig. 3. Questionnaire responses to (a) Q1 and (b) Q2.

suffered for a long time because of intensive drug treatment" (75.0\%). The reason given by the 2 patients in the latter group was: "I believe there is a better form of treatment that was not available when I had my operation."

We then compared various clinical factors among the 3 groups (Table 2). A significantly higher proportion of patients in the earlier group underwent emergency surgery $(p=0.002)$, and the details of the cases in which emergency surgery was performed are shown in Table 3. In the earlier group, the frequency of thiopurine use was nonsignificantly higher and there were more cases of shorter postoperative follow-up observation. The postop- erative SF36 scores are shown in Figure 5. There were no differences between the earlier and appropriate groups for any of the SF36 items, and QOL was maintained at approximately the same level as the Japanese standard values.

\section{Discussion}

Drug treatment forms the basis of treatment for ulcerative colitis, and treatment outcomes have improved in recent years [2]. Some cases will, however, progress 
Fig. 4. Q2: reasons given by patients who responded that they would have preferred to have had surgery earlier (earlier group; multiple answers possible) [1].
Fig. 5. Timing of surgery and SF36 score (norm-based scoring) in the earlier group (would have preferred to have had surgery earlier) and the appropriate group (who felt the timing of their surgery was just right). $\mathrm{PF}$, physical functioning; $\mathrm{RP}$, role physical; BP, bodily pain; $\mathrm{GH}$; general health perceptions; VT, vitality; SF, social functioning; RE, role emotional; $\mathrm{MH}$, mental health.

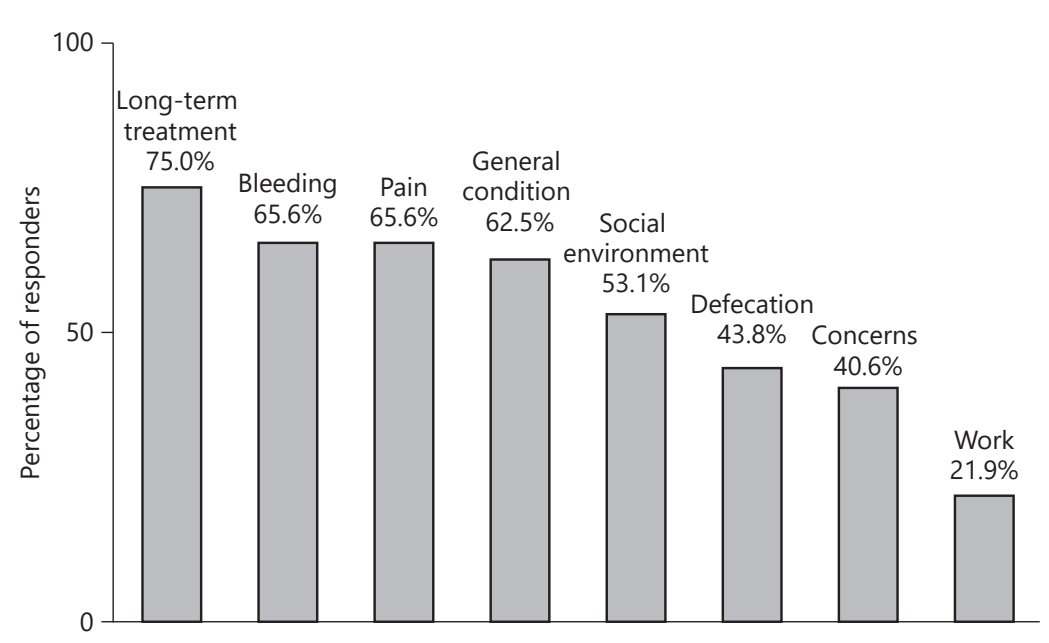

to require surgical treatment due to complications or concomitant onset of colorectal cancer. RP-IPAA was first described in 1978 by Parks and Nicholls [8] as a surgical technique to treat ulcerative colitis, and in the 40 years since then it has become the standard surgical technique for this purpose worldwide [9-12]. In recent years, surgery has commonly been performed after various drug treatments have been attempted, and it is important to ensure appropriate timing of surgery in order to prevent postoperative complications and reduced postoperative QOL. The Japanese patients in this study who underwent RP-IPAA had surgery prior to the development of biologic drugs and so were not taking biologic drugs. When we conducted the survey to determine the timing of surgery and postoperative QOL, we also investigated the patients' perspective of the timing of surgery.

The results of this survey showed that $90.3 \%$ of patients were happy that they underwent surgery. In a study of Japanese patients with inflammatory bowel disease, Ueno et al. [13] reported that only $9.7 \%$ responded that they were dissatisfied or somewhat dissatisfied with hav- 
Table 2. Comparison of clinical factors among patients who responded about the timing of their surgery in relation to preferring surgery was earlier, preferring surgery was later, and believing the timing appropriate

\begin{tabular}{|c|c|c|c|c|}
\hline & $\begin{array}{l}\text { Earlier } \\
(n=32)\end{array}$ & $\begin{array}{l}\text { Appropriate } \\
(n=38)\end{array}$ & $\begin{array}{l}\text { Later } \\
(n=2)\end{array}$ & $p$ value ${ }^{a}$ \\
\hline Men/women, $n$ & $14 / 18$ & $22 / 16$ & $1 / 1$ & 0.337 \\
\hline J-shaped/W-shaped ileal pouch, $n$ & $5 / 27$ & $5 / 33$ & $0 / 2$ & $>0.999$ \\
\hline II/III-stage surgery, $n$ & $9 / 23$ & $10 / 28$ & $0 / 2$ & $>0.999$ \\
\hline Elective surgery/(semi-)emergency surgery, $n$ & $9 / 23$ & $25 / 13$ & $0 / 2$ & 0.002 \\
\hline Relative indication/absolute indication ${ }^{\mathrm{b}}$ & $13 / 16$ & $21 / 10$ & $1 / 1$ & 0.117 \\
\hline Postoperative follow-up observation, months & $73.5(20-348)$ & $140.5(14-307)$ & $106(46-165)$ & 0.011 \\
\hline Period from onset until IPAA, months & $48(1-259)$ & $71(1-300)$ & $63(28-97)$ & 0.157 \\
\hline Total steroid dose, mg & $2,750(0-56,684)$ & $10,637(0-90,000)$ & $1,585(800-2,370)$ & 0.055 \\
\hline Steroid dose/day immediately before surgery, mg & $25(0-120)$ & $20(0-80)$ & $65(60-70)$ & 0.707 \\
\hline Thiopurine use, $n(\%)$ & $8(25.0)$ & $6(15.8)$ & $0(0)$ & 0.381 \\
\hline
\end{tabular}

Values are medians (ranges) unless otherwise indicated. IPAA, ileal pouch anal anastomosis. ${ }^{a}$ Earlier group vs. appropriate group. b Excluding cases of cancer.

Table 3. Comparison of clinical factors among the earlier group and appropriate group in relation to those who underwent emergency surgery

Earlier $(n=23) \quad$ Appropriate $(n=13) \quad p$ value

\footnotetext{
Surgical indication, $n$

Perforation/hemorrhage/toxic megacolon/ severity/fulminant course

Postoperative follow-up observation, months

Period from onset until IPAA, months

Total steroid dose, mg

Preoperative steroid dose/day, mg

Preoperative WBC, $/ \mu \mathrm{L}$

Preoperative CRP, mg/dL

Preoperative Alb, g/dL

Thiopurine use, $n(\%)$

Calcineurin inhibitor use, $n(\%)$

Use of leukocytapheresis, $n$ (\%)
}

$\begin{aligned} & 1 / 3 / 4 / 15 / 0 \\ & 64(20-348) \\ & 26(1-192) \\ & 2,640(180-56,684) \\ & 60(0-120) \\ & 8,395(3,270-16,960) \\ & 1.4(0.15-15.9) \\ & 2.8(2.0-4.3) \\ & 5(21.7) \\ & 2(8.7) \\ & 14(60.9)\end{aligned}$

$\begin{array}{cc}0 / 3 / 1 / 9 / 0 & 0.648 \\ 132(64-307) & \mathbf{0 . 0 0 2} \\ 25(1-122) & 0.818 \\ 4,360(1,540-12,700) & 0.401 \\ 60(20-80) & 0.624 \\ 9,650(4,570-16,270) & 0.473 \\ 1.8(1.0-11.0) & 0.781 \\ 2.7(2.0-4.0) & 0.626 \\ 0(0) & 0.089 \\ 0(0) & 0.402 \\ 6(46.2) & 0.493\end{array}$

Values are medians (ranges) unless otherwise indicated. Alb, albumin; CRP, C-reactive protein; IPAA, ileal pouch anal anastomosis; WBC, white blood cell count.

ing undergone surgery. Lorenzo et al. [6] conducted a questionnaire survey using the Cleveland Global Quality of Life score in the same group of patients 5 and 20 years postoperatively and found a high degree of satisfaction at both time points, with scores of 8.90 and 8.93 of 10 , respectively, for the degree of satisfaction with surgery.
In terms of the timing of surgery, during this study, $44.4 \%$ of patients responded that they would have preferred to have had surgery earlier. Similar responses to those of our Japanese patients were reported by Neumann et al. [1] (53.0\%) and Gröne et al. [9] (43.0\%). 
When we compared the earlier group with the appropriate group, we found that a significantly higher proportion of patients who had undergone emergency surgery responded that they would have preferred to have had surgery earlier. When we investigated the patients who underwent emergency surgery, we found that a nonsignificantly greater number of patients in the earlier group had received thiopurine treatment. The results do not necessarily show that patients with more severe disease would have preferred to have had surgery earlier. However, Biondi et al. [10] discussed the fact that it has become even more difficult to determine the timing of surgery now that the use of biologic drugs has become mainstream practice. The present study did not include any patients who had used biologic drugs before or after surgery, so further studies are required.

It is becoming more common both in Japan and overseas for internal medicine specialists to perform the initial examination and determine the treatment plan $[5,9]$. There are also reports showing a tendency for patients to avoid undergoing surgery due to anxiety about postoperative complications and a lack of information about the surgery $[11,12]$. In our hospital, we have established a cooperative system that includes internal medicine specialists and surgeons who decide on the treatment approach together. Gröne et al. [9] advocate early referral to a surgeon from the early stages of treatment and providing adequate information on surgery to ensure appropriate timing of surgery. In the present study, 2 of the benefits of surgical treatment were indicated by the patients' high degree of postoperative satisfaction and favorable postoperative QOL scores. Several reports from Japan and the United States have indicated similar postoperative QOL results $[1,2,9,14,15]$. However, the present study is limited by the fact that it included only those patients who had undergone surgery without concomitantly taking any biologics, and based solely on the results, it would seem to indicate that surgery should be pursued aggressively. In addition, among the QOL scores, the scores for general health were slightly lower, which complicated the analysis, although the results were similar to those in a report by Røkke et al. [16], which may indicate a certain trend that occurs in postoperative patients.

The limitations of the study were as follows. First, although reference was made to previous studies in terms of the questionnaire that was used, validity was not confirmed. Second, the study was conducted at a single center and involved only patients who had undergone follow-up observation. Third, there was bias in the questionnaire results based on the relationship with the attending physi- cian and participant recall. Also, the questionnaire collection period and observation period were not predefined, and there were changes in medical treatment during the long observation period of the study.

The goal of treatment for ulcerative colitis is to improve patient QOL. Despite the presence of bias in this study, we believe it is clinically significant to investigate the timing of surgery by taking the perspective of Japanese patients into consideration.

\section{Conclusion}

We investigated the timing of surgery for ulcerative colitis from the patients' perspective and found that they were largely satisfied with surgery. However, it is important to consider surgery early for patients who may need emergency surgery.

\section{Acknowledgement}

The authors would like to thank the patients and physicians at Niigata University Medical and Dental Hospital.

\section{Statement of Ethics}

This study was approved by the Niigata University institutional review board (2019-0099).

\section{Disclosure Statement}

The authors have no conflicts of interest to declare.

\section{Funding Sources}

This research received no specific grant from any funding agency in the public, commercial, or not-for-profit sectors.

\section{Author Contributions}

Conception and design of the study: S.Y., H.K., M.N., Y.S., T.W.; acquisition of data: S.Y., H.K., K.A., K.T., H.O., Y.T., M.N., Y.S., J.S., T.W.; analysis and interpretation of data: S.Y., H.K., K.A., K.T., H.O., M.N., Y.S., J.S., T.W.; drafting the manuscript: S.Y., H.K., Y.T., M.N., Y.S., T.W.; study supervision: H.K., J.S., T.W. All authors approved of the final draft submitted. 


\section{References}

1 Neumann PA, Mennigen RB, Senninger N, Bruewer M, Rijcken E. Timing of restorative proctocolectomy in patients with medically refractory ulcerative colitis: the patient's point of view. Dis Colon Rectum. $2012 \mathrm{Jul}$; 55(7):756-61.

2 Windsor A, Michetti P, Bemelman W, Ghosh $\mathrm{S}$. The positioning of colectomy in the treatment of ulcerative colitis in the era of biologic therapy. Inflamm Bowel Dis. 2013 Nov; 19(12):2695-703

3 Xu W, Ye H, Zhu Y, Ding W, Fu J, Cui L, et al. Long-term quality of life associated with early surgical complications in patients with ulcerative colitis after ileal pouch-anal anastomosis: A single-center retrospective study. Int J Surg. 2017 Dec;48:174-9.

4 The Japanese Society of Gastroenterology [Internet]. Evidence-based clinical practice guidelines for inflammatory bowel disease. Tokyo, Japan: Japanese Society of Gastroenterology; 2016 [cited 2019 Dec 2]. p. 74-75. Japanese. Available from: https://www.jsge. or.jp/guideline/guideline/pdf/IBD2016. pdf\#page $=101$

5 Gallo G, Kotze PG, Spinelli A. Surgery in ulcerative colitis: When? How? Best Pract Res Clin Gastroenterol. 2018 Feb-Apr;32-33:71-
6 Lorenzo G, Maurizio C, Maria LP, Tanzanu M, Silvio L, Mariangela $\mathrm{P}$, et al. Ileal pouchanal anastomosis 20 years later: is it still a good surgical option for patients with ulcerative colitis? Int J Colorectal Dis. 2016 Dec; 31(12):1835-43.

7 Andersson P, Söderholm JD. Surgery in ulcerative colitis: indication and timing. Dig Dis. 2009;27(3):335-40.

8 Parks AG, Nicholls RJ. Proctocolectomy without ileostomy for ulcerative colitis. BMJ. 1978 Jul;2(6130):85-8.

9 Gröne J, Lorenz EM, Seifarth C, Seeliger H, Kreis ME, Mueller MH. Timing of surgery in ulcerative colitis in the biologic therapy erathe patient's perspective. Int J Colorectal Dis. 2018 Oct;33(10):1429-35.

10 Biondi A, Zoccali M, Costa S, Troci A, Contessini-Avesani E, Fichera A. Surgical treatment of ulcerative colitis in the biologic therapy era. World J Gastroenterol. 2012 Apr; 18(16):1861-70.

11 Arseneau KO, Sultan S, Provenzale DT, Onken J, Bickston SJ, Foley E, et al. Do patient preferences influence decisions on treatment for patients with steroid-refractory ulcerative colitis? Clin Gastroenterol Hepatol. 2006 Sep; 4(9):1135-42.
12 Thompson KD, Connor SJ, Walls DM, Gollins J, Stewart SK, Bewtra M, et al. Patients with ulcerative colitis are more concerned about complications of their disease than side effects of medications. Inflamm Bowel Dis. 2016 Apr;22(4):940-7.

13 Ueno F, Nakayama Y, Hagiwara E, Kurimoto S, Hibi T. Impact of inflammatory bowel disease on Japanese patients' quality of life: results of a patient questionnaire survey. J Gastroenterol. 2017 May;52(5):555-67.

14 Kameyama H, Iiai T, Shimada Y, Kobayashi Y, Nogami H, Maruyama S, et al. Quality of life after proctocolectomy for ulcerative colitis. Jpn J Gastroenterol Surg. 2010;43(8):77783. Japanese.

15 Watanabe K, Nagao M, Suzuki H, Karasawa $\mathrm{H}$, Ohnuma S, Musha $\mathrm{H}$, et al. The functional outcome and factors influencing the quality of life after ileal pouch anal anastomosis in patients with ulcerative colitis. Surg Today. 2018 Apr;48(4):455-61.

16 Røkke O, Iversen K, Olsen T, Ristesund SM, Eide GE, Turowski GE. Long-term followup with evaluation of the surgical and functional results of the ileal pouch reservoir in restorative proctocolectomy for ulcerative colitis. ISRN Gastroenterol. 2011;2011:625842. 